\title{
A Two-Machine Repair Model with Variable Repair Rate
}

\author{
F.A. van der Duyn Schouten \\ Tilburg University, P.O. Box 90153, 5000 LE Tilburg, The Netherlands and \\ CWI, P.O. Box 4079, 1009 AB Amsterdam, The Netherlands \\ P. Wartenhorst \\ CWI, P.O. Box 4079, 1009 AB Amsterdam, The Netherlands
}

\begin{abstract}
A two-unit cold standby production system with one repairman is considered. After inspection of a failed unit the repairman chooses either a slow or a fast repair rate to carry out the corresponding amount of work. At system breakdown the repairman has an additional opportunity to switch to the fast rate. If there are no fixed costs associated with system breakdowns, then the policy which minimizes longrun average costs is shown to be a two-dimensional control limit rule. If fixed costs are incurred every time the system breaks down, then the optimal policy is not necessarily of control limit type. This is illustrated by a counterexample. Furthermore, we present several performance measures for this maintenance system controlled by a two-dimensional control limit rule. (c) 1993 John Wiley \& Sons, Inc.
\end{abstract}

\section{INTRODUCTION}

In the classical machine repair problem there is a pool of repairmen maintaining a finite number of machines. Since each repairman can serve only one machine at a time, an interference problem exists as soon as the number of machines requiring maintenance exceeds the number of repairmen available. The machine repair problem is also known as machine interference problem and as the finite source queueing problem.

In this article we focus on controlling a production system by adjusting the repair rate. Our system consists of two units and a single repairman. The system is considered to be up if one unit is operating and the other one is either under repair or kept in spare position (cold standby). At breakdown of the operational unit, this unit is sent into the repair facility to be repaired. The spare unit takes over the working position. The system goes down if a unit is still under repair at the breakdown of the other one. Then, at completion of the ongoing repair the repaired unit enters the operating position, a repair is started on the failed unit, and the overall system recovers to up state.

After inspection of a failed unit the repairman knows how much work is to be performed. Then he has the option to choose either a slow or a fast repair 
rate to carry out this amount of work. When the system breaks down while the repairman is working at slow rate, he has an additional opportunity to switch to the fast rate.

In Section 2, we prove that if there are no positive fixed costs associated with overall system breakdowns, then the policy which minimizes the long-run average cost is a two-dimensional control limit rule (also called threshold policy). If fixed costs are incurred every time the system breaks down, then the optimal policy is not necessarily of control limit type. This is illustrated by an example where a four-region policy is shown to be the optimal one. In Section 3, assuming a two-dimensional control limit rule to be imposed on our system, we present explicit expressions from which the long-run average costs, all moments of system up- and downtimes, and an additional number of operational characteristics can be calculated.

An extensive survey of articles on various types of machine repair problems that appeared since the 1976 survey of Pierskalla and Voelker [9] can be found in Cho and Parlar [3]. In many articles structural results are derived on optimal policies such that the long-run average costs are minimized. Systems are controlled, for instance, by reduction of the number of repairmen (e.g., Winston [14], Albright [1]), by opening or closing the repair shop (e.g., Hatoyama [5]), by taking operating units out for preventive maintenance (e.g., Kawai [7], Hatoyama [5]), etc.

Relatively few articles consider direct control of the repair rate of the repairmen (Crabill [4]. Winston [13], Albright [1], Weber and Stidham [12], Karmeshu and Jaiswal [6]). They all assume the repair rate to depend on the number of units that have broken down and are waiting for repair. A general conclusion is that the optimal repair rate is a nondecreasing function of the number of failed units. In these articles, however, failure and repair times are exponentially distributed. fixed costs related to changing the repair rate or with a system breakdown are not considered, and the repair rate is chosen independently of the actual amount of work that is to be performed. In our situation failure and repair times have general probability distribution functions. We also investigate the influence of fixed costs on the structure of the optimal policy. Furthermore, in our model we allow the repair rate to depend on both the number of failed units and on the amount of work to be carried out on the unit that is under repair.

Throughout this article we make the following assumptions.

ASSUMPTION 1: The amount of work that is required to restore a failed unit into as good as new condition is known before the repair is started.

This amount of work becomes known after inspection of the unit. This information is used to decide whether to start a repair at fast or at slow rate. At system breakdown, the residual amount of work is equal to the original amount of work minus the amount of work carried out during operation of the last working unit.

ASSUMPTION 2: It is not possible to switch back to slow rate during a fast repair. 
ASSUMPTION 3: The repairman returns to slow rate at completion of every repair.

Assumptions 2 and 3 are explained by assuming that a regular maintenance crew is present permanently, working at a certain (slow) rate. If necessary, an additional crew is hired to increase the repair rate. The additional crew is hired on a contractual basis for one repair task only. So, even if two consecutive repairs have to be carried out at fast rate, a fixed cost is charged for each of them.

We use the following notation:

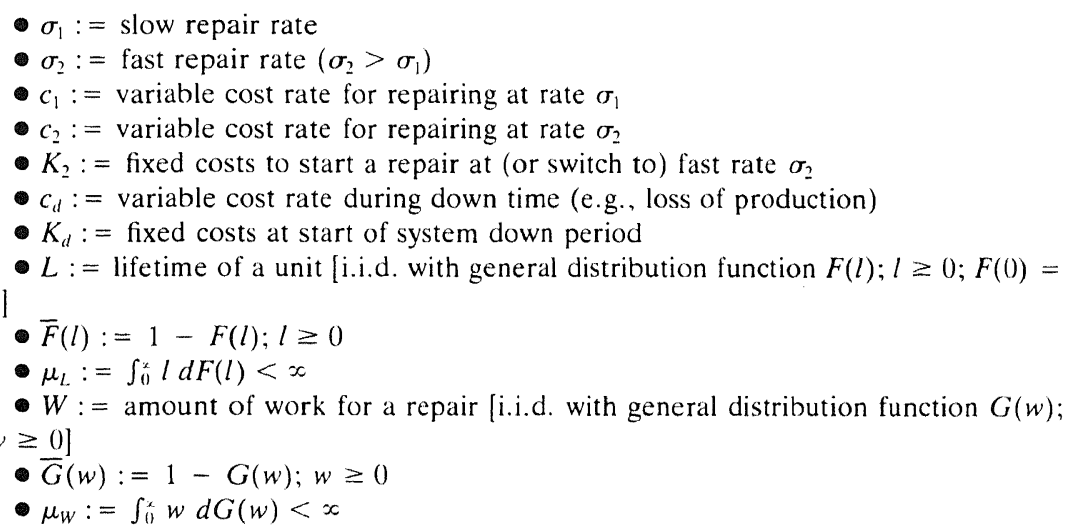

Now we make an additional assumption on the variable down $\operatorname{costs} c_{d}$.

ASSUMPTION 4: There exists a policy with average costs $(A C)$ less than $c_{d}$; i.e., $c_{d}>A C$.

If Assumption 4 is not fulfilled, then either the production system should be closed or the design should be adjusted to make the system profitable.

This article is organized as follows. Section 2 considers the optimal control of the repair unit. First, in Section 2.1, we describe our system by a semi-Markov decision process. In Section 2.2., we give a definition of a control limit rule. Then, in Theorem 2.1, we show that at system breakdown, the repair rate is switched to the fast rate $\sigma_{2}$ according to a control limit rule. In the next two theorems we assume $K_{d}=0$ (no fixed costs at system breakdown). In Theorem 2.2 , we prove that the repair rate to start a repair is chosen according to a control limit rule. For the special case that no additional opportunity exists to switch to $\sigma_{2}$ at system breakdown, in Theorem 2.3, we prove the repair rate to be chosen according to a control limit rule as well. If $K_{d}>0$, then the optimal rate to start a repair is not necessarily chosen according to a control limit rule. This is illustrated by a counterexample in Section 2.3. Assuming a two-dimensional control limit rule to be applied, in Section 3 we present performance measures such as long-run average costs, all moments of system up- and downtimes, system availability, etc. 


\section{OPTIMAL CONTROL}

The repair rates are chosen such that the long-run average costs are minimized. While repairing at slow rate $\sigma_{1}$ (fast rate $\left.\sigma_{2}\right)$, a cost $c_{1}\left(c_{2}\right)$ per unit of time is incurred. Fixed costs $K_{2}$ are charged every time when either a fast rate $\sigma_{2}$ is chosen at the beginning of a repair, or when the repair rate is switched from $\sigma_{1}$ to $\sigma_{2}$ at the beginning of a down period. Due to loss of production an additional variable cost rate $c_{d}$ is incurred during downtime. There are fixed costs $K_{d}$ every time the system breaks down. Lifetimes $(L)$ of the units are i.i.d. according to a general distribution function $F(l) ; l \geq 0$ with finite mean $\mu_{L}$. The amounts of work that have to be performed on failed units form a sequence of i.i.d. random variables with general distribution function $G(w) ; w \geq 0$ with finite mean $\mu_{W}$. Repaired units are as good as new.

\subsection{Semi-Markov Decision Process}

The system is inspected at two types of decision epochs: Either when a new unit enters operation and a repair is started on the other one, or when the operating unit just failed and repair on the other unit has not been finished yet (system breakdown). Inspection reveals the system to be in a state $x \in \mathscr{x}$. The infinite state space,$Z$ is defined by

$$
x:=\left\{(U, w),\left(D, \sigma_{1}, w\right),\left(D, \sigma_{2}, w\right) ; w \geq 0\right\},
$$

where

\footnotetext{
$U(D)$ denotes that a new repair is to be started (an ongoing repair has to be continued at system breakdown),

$\sigma_{1}, \sigma_{2}$ denotes the current repair rate,

$w$ denotes the amount of work (still) to be carried out on the unit currently under repair.
}

If a new repair is started, then after inspection the repairman finds the system in a state $(U, w)$ and has to choose either a slow or a fast rate to perform this repair. If the repairman is working at slow rate, then at system breakdown he has to decide to continue working at slow rate or to switch to fast rate. Formally, if at an inspection epoch the system is in state $x \in \mathscr{X}$, then the repairman has to choose an action $\sigma \in A(x)$. The finite action space $A(x)$ is given by

$$
A(x)= \begin{cases}\left\{\sigma_{1}, \sigma_{2}\right\}, & \text { if } x \in\left\{(U, w),\left(D, \sigma_{1}, w\right) ; w \geq 0\right\}, \\ \sigma_{2}, & \text { if } x \in\left\{\left(D, \sigma_{2}, w\right) ; w \geq 0\right\},\end{cases}
$$

where $\sigma_{1}, \sigma_{2}$ denotes the rate at which the current (residual) repair will be continued.

A stationary policy $\pi$ is employed; i.e., the repair rate chosen depends on the present state of the system only:

$$
\pi(x) \in A(x) ; \quad x \in \mathscr{X} .
$$


This controlled dynamic system is a semi-Markov decision process because the following properties are satisfied (cf. Tijms [11]). The time until, and the state at, the next decision epoch depend only on the present state $x \in \mathscr{X}^{\prime}$ and the chosen action $\sigma \in A(x)$, and are thus independent of the past history of the system. Also, the costs incurred until the next decision epoch depend only on the present state and the action chosen in that state. Let

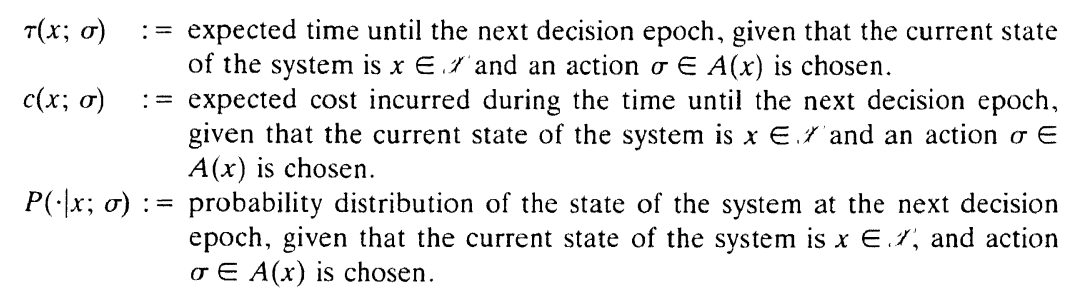

In Appendix A we show that, in case $G(\cdot)$ has finite support, there exists a bounded function $v(x), x \in \mathscr{X}$ and a constant $g$, which satisfy the following set of average cost optimality equations:

$$
v(x)=\min _{\sigma \in A(x)}\left\{c(x ; \sigma)-g \tau(x ; \sigma)+\int_{y \in,} v(y) d P(y \mid x ; \sigma)\right\} ; \quad x \in \mathscr{x} .
$$

According to Ross [10], for any policy which, when in state $x$, selects an action minimizing the right-hand side of (3), we have that the long-run average costs are minimized and are equal to $g$. So to find the optimal policy $\pi^{*}$ we have to investigate which decision is to be made such that (3) is minimized.

In our model $\tau(x ; \sigma)$ and $c(x ; \sigma)$ are given by the following expressions:

$$
\begin{aligned}
\tau\left(D, \sigma_{1}, w ; \sigma_{1}\right) & =\frac{w}{\sigma_{1}}, \\
\tau\left(D, \sigma_{1}, w ; \sigma_{2}\right) & =\frac{w}{\sigma_{2}}, \\
\tau\left(D, \sigma_{2}, w ; \sigma_{2}\right) & =\frac{w}{\sigma_{2}}, \\
\tau\left(U, w ; \sigma_{1}\right) & =\mu_{L}, \\
\tau\left(U, w ; \sigma_{2}\right) & =\mu_{L}, \\
c\left(D, \sigma_{1}, w ; \sigma_{1}\right) & =K_{d}+\left(c_{1}+c_{d}\right) \frac{w}{\sigma_{1}}, \\
c\left(D, \sigma_{1}, w ; \sigma_{2}\right) & =K_{d}+K_{2}+\left(c_{2}+c_{d}\right) \frac{w}{\sigma_{2}},
\end{aligned}
$$




$$
\begin{aligned}
c\left(D, \sigma_{2}, w ; \sigma_{2}\right) & =K_{d}+\left(c_{2}+c_{d}\right) \frac{w}{\sigma_{2}}, \\
c\left(U, w ; \sigma_{1}\right) & =c_{1}\left[\int_{0}^{w / \sigma_{1}} l d F(l)+\frac{w}{\sigma_{1}} \bar{F}\left(\frac{w}{\sigma_{1}}\right)\right] \\
& =c_{1}\left[\frac{w}{\sigma_{1}}-\int_{0}^{w / \sigma_{1}} F(t) d t\right], \\
c\left(U, w ; \sigma_{2}\right) & =K_{2}+c_{2}\left[\frac{w}{\sigma_{2}}-\int_{0}^{w / \sigma_{2}} F(t) d t\right] .
\end{aligned}
$$

Substituting these expressions for $\tau(x ; \sigma)$ and $c(x ; \sigma)$ into (3) gives us the optimality equations for our semi-Markov decision process:

$$
\begin{aligned}
& v(U, w)=\min \left\{c_{1}\left[\frac{w}{\sigma_{1}}-\int_{0}^{w / \sigma_{1}} F(t) d t\right]\right. \\
& +\int_{0}^{w / \sigma_{1}} v\left(D, \sigma_{1}, w-\sigma_{1} l\right) d F(l) \\
& +\bar{F}\left(\frac{w}{\sigma_{1}}\right) Z, K_{2}+c_{2}\left[\frac{w}{\sigma_{2}}-\int_{0}^{w / \sigma_{2}} F(t) d t\right] \\
& \left.+\int_{0}^{w / \sigma_{2}} v\left(D, \sigma_{2}, w-\sigma_{2} l\right) d F(l)+\bar{F}\left(\frac{w}{\sigma_{2}}\right) Z\right\}-g \mu_{L}, \\
& v\left(D, \sigma_{1}, w\right)=\min \left\{\left(c_{1}+c_{d}-g\right) \frac{w}{\sigma_{1}}, K_{2}+\left(c_{2}+c_{d}-g\right) \frac{w}{\sigma_{2}}\right\} \\
& +K_{d}+Z \text {, } \\
& v\left(D, \sigma_{2}, w\right)=\left(c_{2}+c_{d}-g\right) \frac{w}{\sigma_{2}}+K_{d}+Z,
\end{aligned}
$$

where

$$
Z:=\int_{0}^{\infty} v(U, w) d G(w)
$$

\subsection{Control Limit Rule}

From (1) we observe that there are two types of decision epochs in which an actual decision should be made: $(U, w)$ and $\left(D, \sigma_{1}, w\right)$. [Due to Assumption 2 
in $\left(D, \sigma_{2}, w\right)$ the repair rate remains unchanged.] For any stationary policy $\pi$. we define [cf. (2)]

$$
\begin{aligned}
& \pi_{L^{\prime}}(w):=\pi\left(U, w^{\prime}\right), \\
& \pi_{D}(w):=\pi\left(D, \sigma_{1}, w\right) .
\end{aligned}
$$

DEFINITION 2.1: For any stationary policy $\pi$, we call $\pi_{l}$ a control limit rule $\operatorname{CLR}\left(m_{U}\right)$ if there exists some threshold value $m_{l}$, such that

$$
\pi_{U}(w)=\sigma_{2}, \quad \text { iff } w>m_{l} .
$$

For $\pi_{D}$ a similar definition of $\operatorname{CLR}\left(m_{D}\right)$ applies. If $\pi_{U}$ is $\operatorname{CLR}\left(m_{U}\right)$ and $\pi_{D}$ is $\operatorname{CLR}\left(m_{D}\right)$ then the overall system is controlled by a two-dimensional control limit rule $\operatorname{CLR}\left(m_{U}, m_{D}\right)$. The optimal policy is denoted by $\pi^{*}$ with corresponding $\pi_{U}^{*}$ and $\pi_{D}^{*}$.

In Theorem 2.1, we prove that in state $\left(D, \sigma_{1}, w\right)$ the repair rate is switched from $\sigma_{1}$ to $\sigma_{2}$ according to a control limit rule $\operatorname{CLR}\left(m_{D}\right)$, no matter what (stationary) policy is followed in states $(U, w)$. For $K_{d}=0$, and using the result of Theorem 2.1, we prove in Theorem 2.2 that in state $(U, w)$ the fast rate $\sigma_{2}$ is chosen according to a control limit rule $\operatorname{CLR}\left(m_{U}\right)$ as well. In Theorem 2.3, we consider a restricted version of the model presented so far. In this adapted version we assume that it is not possible to change the repair rate during an ongoing repair. This means that in state $(U, w)$ a repair rate is chosen which holds for the entire repair task. For such situations with $K_{d}=0$, in Theorem 2.3 again we prove that the optimal rate is chosen according to a CLR. However, if $K_{d}>0$ then the optimal policy in state $(U, w)$ is not necessarily a CLR. A counterexample and an intuitive explanation are given in Section 2.3.

THEOREM 2.1: $\pi_{D}^{*}$ is a control limit rule $\operatorname{CLR}\left(m_{D}\right)$.

PROOF: From (5) we conclude that $\sigma_{2}$ is chosen in state $\left(D, \sigma_{1}, w\right)$ iff

$$
\begin{aligned}
& K_{2}+\left(c_{2}+c_{d}-g\right) \frac{w}{\sigma_{2}}<\left(c_{1}+c_{d}-g\right) \frac{w}{\sigma_{1}} \\
& \Leftrightarrow w\left[\frac{c_{1}+c_{d}-g}{\sigma_{1}}-\frac{c_{2}+c_{d}-g}{\sigma_{2}}\right]>K_{2} .
\end{aligned}
$$

If

$$
\frac{c_{1}+c_{d}-g}{\sigma_{1}} \leq \frac{c_{2}+c_{d}-g}{\sigma_{2}},
$$

then (7) will not hold for any $w \geq 0$. So, the repairman will never switch to $\sigma_{2}$ $\left(m_{D}:=x\right)$. If

$$
\frac{c_{1}+c_{d}-g}{\sigma_{1}}>\frac{c_{2}+c_{d}-g}{\sigma_{2}},
$$


then $\sigma_{2}$ is chosen iff

$$
w>m_{D},
$$

where

$$
m_{D}:=K_{2} /\left[\frac{c_{1}+c_{d}-g}{\sigma_{1}}-\frac{c_{2}+c_{d}-g}{\sigma_{2}}\right] .
$$

So, $\sigma_{2}$ is chosen according to a control limit rule.

REMARK 2.1: In Theorem 2.1 we have not used any information about the structure of the (stationary) policy employed in state $(U, w)$. Actually, assuming an arbitrary stationary policy to be employed in state $(U, w)$, one can construct an alternative semi-Markov decision process on the embedded states $\left\{(D, \sigma, w) ; w>0, \sigma \in\left\{\sigma_{1}, \sigma_{2}\right\}\right\}$ only. From the corresponding optimality equations it is easy to see that the conditions for the repairman to choose $\sigma_{2}$ in state $\left(D, \sigma_{1}, w\right)$ are similar to those found in the proof of Theorem 2.1. So, in state $\left(D, \sigma_{1}, w\right)$ the repair rate is switched from $\sigma_{1}$ to $\sigma_{2}$ according to a control limit rule $\operatorname{CLR}\left(m_{D}\right)$, no matter what stationary policy is followed in state $(U, w)$.

In Theorem 2.1 we have shown that the best way of switching to $\sigma_{2}$ at the beginning of a down period, is according to a CLR. Therefore, in Theorem 2.2 we assume $\pi_{D}^{*}$ to be $\operatorname{CLR}\left(m_{D}\right)$, where $m_{D}$ is the optimal control limit in state $\left(D, \sigma_{1}, w\right)$, as defined by $(8)$.

THEOREM 2.2: If $K_{d}=0$ and either $c_{1} / \sigma_{1} \geq c_{2} / \sigma_{2}$ or $F(l)$ is IFR, then $\pi_{U}^{*}$ is a control limit rule $\operatorname{CLR}\left(m_{U}\right)$.

PROOF: Consider the optimality equations (4)-(6) with $K_{d}=0$. First we substitute (6) into (4). By changing the order of integration, this simplifies the second minimization term of (4):

$$
\begin{aligned}
K_{2}+c_{2}\left[\frac{w}{\sigma_{2}}-\int_{0}^{w / \sigma_{2}} F(t) d t\right] & +\int_{0}^{w / \sigma_{2}} v\left(D, \sigma_{2}, w-\sigma_{2} l\right) d F(l)+\bar{F}\left(\frac{w}{\sigma_{2}}\right) Z \\
& =K_{2}+\frac{c_{2}}{\sigma_{2}} w+\left(c_{d}-g\right) \int_{0}^{w / \sigma_{2}} F(t) d t+Z .
\end{aligned}
$$

For the remainder of this proof we distinguish three cases.

Case (i):

$$
\frac{c_{1}+c_{d}-g}{\sigma_{1}} \leq \frac{c_{2}+c_{d}-g}{\sigma_{2}} .
$$

From the proof of Theorem 2.1 we know that in this case in state $\left(D, \sigma_{1}, w\right)$ always $\sigma_{1}$ is chosen. In $(U, w)$ there is even less reason to work at fast rate, as 
no variable down costs $c_{d}$ are accounted (initially) and $K_{d}=0$. So, one would expect $\sigma_{1}$ to be optimal in this case. This is just what happens. From (5) we see

$$
v\left(D, \sigma_{1}, w\right)=\left(c_{1}+c_{d}-g\right) \frac{w}{\sigma_{1}}+Z .
$$

Substitution of (10) into (4), and using (9) yields

$$
\begin{aligned}
v(U, w)=\min _{\sigma_{1}, \sigma_{2}}\left\{\frac{c_{1}}{\sigma_{1}} w+\left(c_{d}-g\right) \int_{0}^{w / \sigma_{1}} F(t) d t\right. \\
\left.K_{2}+\frac{c_{2}}{\sigma_{2}} w+\left(c_{d}-g\right) \int_{0}^{w / \sigma_{2}} F(t) d t\right\}+Z-g \mu_{L} .
\end{aligned}
$$

Now $\sigma_{2}$ is chosen iff

$$
\begin{aligned}
& K_{2}+\frac{c_{2}}{\sigma_{2}} w+\left(c_{d}-g\right) \int_{0}^{w / \sigma_{2}} F(t) d t<\frac{c_{1}}{\sigma_{1}} w+\left(c_{d}-g\right) \int_{0}^{w / \sigma_{1}} F(t) d t \\
& \Leftrightarrow\left(c_{d}-g\right) \int_{w / \sigma_{2}}^{w / \sigma_{1}} F(t) d t+\left[\frac{c_{1}}{\sigma_{1}}-\frac{c_{2}}{\sigma_{2}}\right] w>K_{2} .
\end{aligned}
$$

However,

$$
\begin{aligned}
\left(c_{d}-g\right) \int_{w / \sigma_{2}}^{w / \sigma_{1}} F(t) d t+\left[\frac{c_{1}}{\sigma_{1}}-\right. & \left.\frac{c_{2}}{\sigma_{2}}\right] w \\
& \leq\left[\frac{c_{1}+c_{d}-g}{\sigma_{1}}-\frac{c_{2}+c_{d}-g}{\sigma_{2}}\right] w \leq 0 .
\end{aligned}
$$

By combining (12) and (13) we conclude that $\sigma_{1}$ is chosen for all $w \geq 0\left(m_{U}=\right.$ $\infty)$.

Case (ii):

$$
\frac{c_{1}+c_{d}-g}{\sigma_{1}}>\frac{c_{2}+c_{d}-g}{\sigma_{2}} \text { and } w \leq m_{D}
$$

As in Case (i), we note that if condition (ii) holds in a down state $\left(D, \sigma_{1}, w\right)$, then $\sigma_{1}$ is chosen (due to Theorem 2.1). Again we prove that $\sigma_{1}$ is optimal in $(U, w)$ as well, which is intuitively clear. In (4) $v\left(D, \sigma_{1}, w-\sigma_{1} l\right)$ only occurs for

$$
0 \leq w-\sigma_{1} l \leq w \leq m_{D}
$$


So, again (10) is to be substituted into (4). From (11) we see that $\sigma_{2}$ is chosen if (12) holds. However,

$$
\begin{aligned}
\left(c_{d}-g\right) \int_{w / \sigma_{2}}^{w / \sigma_{1}} F(t) d t+\left[\frac{c_{1}}{\sigma_{1}}-\frac{c_{2}}{\sigma_{2}}\right] w \\
\quad \leq\left[\frac{c_{1}+c_{d}-g}{\sigma_{1}}-\frac{c_{2}+c_{d}-g}{\sigma_{2}}\right] m_{D}=K_{2},
\end{aligned}
$$

by (8). Now again by combining (12) and (14) we conclude that $\sigma_{1}$ is chosen for all $w \geq 0$.

Case (iii)

$$
\frac{c_{1}+c_{d}-g}{\sigma_{1}}>\frac{c_{2}+c_{d}-g}{\sigma_{2}} \text { and } w>m_{D}
$$

In this situation a nontrivial control limit is to be expected. Furthermore in this case we will actually use the condition that either $c_{1} / \sigma_{1} \geq c_{2} / \sigma_{2}$ or $F(l)$ is IFR. From Theorem 2.1 we know that $\sigma_{2}$ is chosen in state $\left(D, \sigma_{1}, w-\sigma_{1} l\right)$ iff

$$
w-\sigma_{1} l>m_{D} \Leftrightarrow 0 \leq l<\left(w-m_{D}\right) / \sigma_{1},
$$

and $\sigma_{1}$ is chosen otherwise. We use this result to simplify the first minimization term in (4):

$$
\begin{aligned}
& \int_{0}^{w / \sigma_{1}} v\left(D, \sigma_{1}, w-\sigma_{1} l\right) d F(l)+\bar{F}\left(\frac{w}{\sigma_{1}}\right) Z \\
& =\int_{0}^{\left(w-m_{D}\right) / \sigma_{1}}\left[K_{2}+\left(c_{2}+c_{d}-g\right) \frac{w-\sigma_{1} l}{\sigma_{2}}+Z\right] d F(l) \\
& \quad+\int_{\left(w-m_{D}\right) / \sigma_{1}}^{w / \sigma_{1}}\left[\left(c_{1}+c_{d}-g\right) \frac{w-\sigma_{1} l}{\sigma_{1}}+Z\right] d F(l)+\bar{F}\left(\frac{w}{\sigma_{1}}\right) Z \\
& =\left(c_{2}+c_{d}-g\right) \frac{\sigma_{1}}{\sigma_{2}} \int_{0}^{\left(w-m_{D}\right) / \sigma_{1}} F(t) d t \\
& \quad+\left(c_{1}+c_{d}-g\right) \int_{\left(w-m_{D}\right) / \sigma_{1}}^{w / \sigma_{1}} F(t) d t+Z .
\end{aligned}
$$

After substitution of (9) and (15) into (4) we finally find that $\sigma_{2}$ is chosen iff

$$
K(w)>C H(w),
$$


where

$$
\begin{aligned}
K(w) & :=\int_{0}^{\left(w-m_{D}\right) / \sigma_{1}}[1-F(t)] d t, \\
H(w) & :=\int_{w / \sigma_{2}}^{w / \sigma_{1}}[1-F(t)] d t, \\
C & :=\left(c_{d}-g\right) /\left(\sigma_{1}\left[\frac{c_{1}+c_{d}-g}{\sigma_{1}}-\frac{c_{2}+c_{d}-g}{\sigma_{2}}\right]\right) .
\end{aligned}
$$

Due to Assumption $4 c_{d}>g$, and thus $C>0$. From (16), it is easy to see that the optimal policy is CLR if $0<C \leq 1$. We have not been able, however, to derive a similar general result for $C>1$ without the additional assumptions of the theorem. Furthermore, since $C$ contains $g$, the condition $0<C \leq 1$ cannot be checked beforehand, which makes it useless for practical purposes.

To obtain sufficient conditions that guarantee the optimality of a CLR, we note that

$$
\begin{gathered}
K\left(m_{D}\right)=0 ; \quad \lim _{w \rightarrow \infty} K(w)=\mu_{L}>0, \\
C H\left(m_{D}\right)=C \int_{m_{D} / \sigma_{2}}^{m_{D} / \sigma_{1}}[1-F(t)] d t \geq 0 ; \quad \lim _{w \rightarrow \infty} C H(w)=0 .
\end{gathered}
$$

So,

$$
K\left(m_{D}\right) \leq C H\left(m_{D}\right) ; \quad K(\infty)>C H(\infty)
$$

This implies that $K(w)$ and $C H(w)$ intersect at least once; $K(w)$ lies below $C H(w)$ initially and exceeds $C H(w)$ finally. A first conclusion from (18) is that there exists some $0 \leq \bar{w}<\infty$ such that $\sigma_{2}$ is chosen for all $w>\bar{w}$.

From (16) and (18) we see that a necessary and sufficient condition for a CLR to be optimal is that $K(w)$ and $C H(w)$ intersect only once. A sufficient (not necessary) condition to guarantee this is

$$
K^{\prime}(w) \geq C H^{\prime}(w), \quad \text { for } w>S
$$

where $K^{\prime}(w)$ and $H^{\prime}(w)$ denote the derivatives of $K(w)$ and $H(w)$, respectively:

$$
\begin{aligned}
& K^{\prime}(w):=\frac{1}{\sigma_{1}}\left[1-F\left(\frac{w-m_{D}}{\sigma_{1}}\right)\right] \geq 0 \\
& H^{\prime}(w):=\frac{1}{\sigma_{1}}\left[1-F\left(\frac{w}{\sigma_{1}}\right)\right]-\frac{1}{\sigma_{2}}\left[1-F\left(\frac{w}{\sigma_{2}}\right)\right] \in \mathbb{R},
\end{aligned}
$$


and

$$
S:=\text { first intersection point; } \quad K(S)=C H(S) ; \quad K^{\prime}(S)>C H^{\prime}(S) .
$$

A sufficient condition which guarantees (19) is

$$
\frac{c_{1}}{\sigma_{1}} \geq \frac{c_{2}}{\sigma_{2}}
$$

From (21) we see that in this case

$$
\begin{aligned}
& C H^{\prime}\left(w^{\prime}\right) \leq C\left[\frac{1}{\sigma_{1}}-\frac{1}{\sigma_{2}}\right]\left[1-F\left(\frac{w-m_{D}}{\sigma_{1}}\right)\right] \\
&=\left[\frac{c_{1}+c_{d}-g}{\sigma_{1}}-\frac{c_{2}+c_{d}-g}{\sigma_{2}}\right]^{-1} \\
& \times\left[\frac{c_{d}-g}{\sigma_{1}}-\frac{c_{d}-g}{\sigma_{2}}\right] \frac{1}{\sigma_{1}}\left[1-F\left(\frac{w-m_{D}}{\sigma_{1}}\right)\right] \leq K^{\prime}(w) .
\end{aligned}
$$

So, if (22) holds then the optimal policy is CLR.

Another condition sufficient for (19) to hold is that the lifetime distribution function with density function $f(l)$ is IFR; i.e., $F(l)$ has an increasing failure rate:

$$
\frac{f(x)}{1-F(x)} \leq \frac{f(y)}{1-F(y)}, \quad \text { for } x<y
$$

which is explained as follows. From (17) and (21) we see

$$
H(0)=0 ; \quad H^{\prime}(0)>0 ; \quad H(\infty)=0 .
$$

In Lemma B.1 we prove that if $F(l)$ is IFR, then $H(w)$ is unimodal; i.e., $H(w)$ reaches its maximum in $w_{H}^{*}$ (say), and

$$
\begin{array}{ll}
H^{\prime}(w)>0, & \text { for } 0 \leq w<w_{H}^{*}, \\
H^{\prime}(w)<0, & \text { for } w>w_{H}^{*} .
\end{array}
$$

If $S \geq w_{H}^{*}$ then (19) is certainly satisfied because $H^{\prime}(w)<0$ for $w>S$. If $S<$ $w_{H}^{*}$ then consider

$$
L(w):=\frac{K^{\prime}(w)}{H^{\prime}(w)}, \quad \text { for } S<w<w_{H}^{*}
$$


Now (19) is satisfied if

$$
L(w)>C, \quad \text { for } S<w<w_{H}^{*} .
$$

A sufficient condition to guarantee (24) is for $S<w<w_{H}^{*}$ :

$L^{\prime}(w) \geq 0$

$$
\Leftrightarrow \frac{\left(1 / \sigma_{1}\right) f\left[\left(w-m_{D}\right) / \sigma_{1}\right]}{1-F\left[\left(w-m_{D}\right) / \sigma_{1}\right]} \leq \frac{\left(1 / \sigma_{1}^{2}\right) f\left(w / \sigma_{1}\right)-\left(1 / \sigma_{2}^{2}\right) f\left(w / \sigma_{2}\right)}{1 / \sigma_{1}\left[1-F\left(w / \sigma_{1}\right)\right]-1 / \sigma_{2}\left[1-F\left(w / \sigma_{2}\right)\right]},
$$

which is satisfied if $F(l)$ is IFR. Thus (19) is satisfied, which is a sufficient condition for a CLR to be optimal.

In Theorem 2.2, the condition $c_{1} / \sigma_{1} \geq c_{2} / \sigma_{2}$ has an intuitively appealing interpretation. Repairing at rate $\sigma_{i}$ costs $c_{i}$ per unit of time, while $\sigma_{i}$ units of work are performed per unit of time $(i=1,2)$. Thus $c_{i} / \sigma_{i}$ denotes the cost of performing one unit of work at rate $\sigma_{i}$. If $c_{1} / \sigma_{1} \geq c_{2} / \sigma_{2}$ then working at fast rate $\sigma_{2}$ reduces both variable repair costs and the expected length of a down period. Once $w$ is so large that $K_{2}$ is sufficiently compensated by these reductions, there is no reason to believe $\sigma_{1}$ to become optimal again for larger values of $w$.

The case $c_{1} / \sigma_{1}<c_{2} / \sigma_{2}$ is less evident, because working at slow rate is cheapest. Both $K_{2}$ and the additional variable repair costs have to be fully compensated by a reduction of the expected downtime. Once $w$ and $\sigma$ are given, the expected downtime is determined by the lifetime distribution $F(l)$. Theorem 2.2 states that $\pi_{U}^{*}$ is a control limit rule if $F(l)$ is IFR, which is intuitively clear. We have not been able, however, to develop intuitive arguments to explain why a control limit rule would not be optimal if both $c_{1} / \sigma_{1}<c_{2} / \sigma_{2}$ and $F(l)$ is non-IFR.

Theorem 2.3 considers a restricted model where the repair rate cannot be switched during an ongoing repair. This is equivalent with setting $m_{D}=\infty$ in the general model considered in Theorems 2.1 and 2.2. For this restricted model we prove in Theorem 2.3 that $\pi_{U}^{*}$ is a CLR, without any limitations on either $F(l)$ or the variable cost rates.

Since the repair rate remains unchanged at system breakdown, we have to consider embedded states $(U, w)$ only. Thus we have to consider a modified semi-Markov decision process with corresponding state space $x$ 'and action space $A(x)$ given by

$$
\begin{aligned}
\mathscr{X}: & =\{w ; w \geq 0\}, \\
A(x) & :=\left\{\sigma_{1}, \sigma_{2}\right\}, \quad x \in \mathscr{X} .
\end{aligned}
$$

$\tau(x ; \sigma)$ and $c(x ; \sigma)$ are given by

$$
\begin{aligned}
\tau\left(w ; \sigma_{1}\right) & =\int_{0}^{w / \sigma_{1}} \frac{w}{\sigma_{1}} d F(l)+\int_{w / \sigma_{1}}^{\infty} l d F(l) \\
& =\frac{w}{\sigma_{1}}+\int_{w / \sigma_{1}}^{\infty} \bar{F}(t) d t
\end{aligned}
$$




$$
\begin{aligned}
\tau\left(w ; \sigma_{2}\right) & =\frac{w}{\sigma_{2}}+\int_{w / \sigma_{2}}^{\infty} \bar{F}(t) d t \\
c\left(w ; \sigma_{1}\right) & =c_{1} \frac{w}{\sigma_{1}}+K_{d} F\left(\frac{w}{\sigma_{1}}\right)+c_{d} \int_{0}^{w / \sigma_{1}}\left(\frac{w}{\sigma_{1}}-l\right) d F(l) \\
& =c_{1} \frac{w}{\sigma_{1}}+K_{d} F\left(\frac{w}{\sigma_{1}}\right)+c_{d} \int_{0}^{w / \sigma_{1}} F(t) d t \\
c\left(w ; \sigma_{2}\right) & =K_{2}+c_{2} \frac{w}{\sigma_{2}}+K_{d} F\left(\frac{w}{\sigma_{2}}\right)+c_{d} \int_{0}^{w / \sigma_{2}} F(t) d t .
\end{aligned}
$$

As in the general case, it can be shown that for this model there exist $v(x)$, $x \in, l$ and $g$ which satisfy the average cost optimality equations [cf. (3)]:

$$
\begin{aligned}
v(w)= & \min \left\{\left(c_{1}-g\right) \frac{w}{\sigma_{1}}+K_{d} F\left(\frac{w}{\sigma_{1}}\right)+c_{d} \int_{0}^{w / \sigma_{1}} F(t) d t\right. \\
& -g \int_{w / \sigma_{1}}^{\infty} \bar{F}(t) d t, K_{2}+\left(c_{2}-g\right) \frac{w}{\sigma_{2}}+K_{d} F\left(\frac{w}{\sigma_{2}}\right) \\
& \left.+c_{d} \int_{11}^{w / \sigma_{2}} F(t) d t-g \int_{w / \sigma_{2}}^{\infty} \bar{F}(t) d t\right\} \\
& +\int_{0}^{\infty} v(w) d G(w) .
\end{aligned}
$$

THEOREM 2.3: If $K_{d}=0$ then in the restricted model $\pi_{U}^{*}$ is a control limit rule $\operatorname{CLR}\left(m_{U}\right)$.

PROOF: If $K_{d}=0$ then from (25) we see that $\sigma_{1}$ is chosen if

$$
\begin{aligned}
& K_{2}+\left(c_{2}+c_{d}-g\right) \frac{w}{\sigma_{2}}-c_{d} \int_{0}^{w / \sigma_{2}}[1-F(t)] d t-g \int_{w / \sigma_{2}}^{\infty}[1-F(t)] d t \\
& \geq\left(c_{1}+c_{d}-g\right) \frac{w}{\sigma_{1}}-c_{d} \int_{0}^{w / \sigma_{1}}[1-F(t)] d t-g \int_{w / \sigma_{1}}^{\infty}[1-F(t)] d t \\
& \Leftrightarrow K_{2}-\left[\frac{c_{1}+c_{d}-g}{\sigma_{1}}-\frac{c_{2}+c_{d}-g}{\sigma_{2}}\right] w \\
& +\left(c_{d}-g\right) \int_{w / \sigma_{2}}^{w / \sigma_{1}}[1-F(t)] d t \geq 0 \Leftrightarrow H(w) \geq \bar{C} w-\bar{K},
\end{aligned}
$$


where $H(w)$ is defined by (17) and

$$
\begin{aligned}
& \bar{C}:=\left[\frac{c_{1}+c_{d}-g}{\sigma_{1}}-\frac{c_{2}+c_{d}-g}{\sigma_{2}}\right]\left(c_{d}-g\right)^{-1}, \\
& \bar{K}:=K_{2} /\left(c_{d}-g\right) .
\end{aligned}
$$

Due to Assumption $4 \bar{K}>0$. In Lemma B.2 (Appendix B), it is shown that

$$
H(\alpha w) \geq \alpha H(w), \quad \text { for } w \geq 0 \text { and } 0 \leq \alpha \leq 1 .
$$

Now suppose (26) is satisfied for some $m_{U} \geq 0$; i.e., $\sigma_{1}$ is chosen if $w=m_{U}$. Then

$$
H\left(m_{U}\right) \geq \bar{C} m_{U}-\bar{K}
$$

Then using Lemma B.2, for $0 \leq \alpha \leq 1$ :

$$
H\left(\alpha m_{U}\right) \geq \alpha H\left(m_{U}\right) \geq \alpha\left(\bar{C} m_{U}-\bar{K}\right) \geq \bar{C} \alpha m_{U}-\bar{K} .
$$

Thus,

$$
\forall 0 \leq w \leq m_{U}, \quad H(w) \geq \bar{C} w-\bar{K} .
$$

So, if $\sigma_{1}$ is the optimal repair rate for some $m_{U} \geq 0$, then $\sigma_{1}$ is the optimal choice for all $0 \leq w \leq m_{U}$. This is exactly the definition of a control limit rule (cf. Definition 2.1).

\subsection{Counterexample: Four-Region Policy}

If $K_{d}>0$ then the optimal policy in $(U, w)$ is not always a control limit rule. It may be a four-region $\left(m_{1}, m_{2}, m_{3}\right)$ policy which is defined as follows:

$$
\begin{aligned}
& \text { if } w \in\left\{\left(0, m_{1}\right] \cup\left(m_{2}, m_{3}\right]\right\} \text {, then } \sigma_{1} \text { is chosen, } \\
& \text { if } w \in\left\{\left(m_{1}, m_{2}\right] \cup\left(m_{3}, \infty\right)\right\} \text {, then } \sigma_{2} \text { is chosen, }
\end{aligned}
$$

where

$$
0 \leq m_{1} \leq m_{2} \leq m_{3}<\infty .
$$

The optimality of this four-region policy is intuitively argued as follows. If $w \in\left(0, m_{1}\right]$ then the probability of a system breakdown is negligible. So, there is no need to switch to the fast rate $\sigma_{2}$. If $w \in\left(m_{1}, m_{2}\right]$ there may be a considerable chance of a system breakdown occurring during a slow repair, incurring fixed costs $K_{d}$. One may prevent the system from breaking down by switching to $\sigma_{2}$. Then the expected down costs are reduced such that $K_{2}$ is sufficiently compensated. If $w \in\left(m_{2}, m_{3}\right]$ then there may be a considerable chance of a system 
breakdown, even when repairing at fast rate. So, in this case $K_{2}$ has to be compensated mostly by a reduction of variable repair and down costs. If this reduction is too small then $\sigma_{1}$ becomes optimal again. For $w>m_{3}$ the reduction of variable costs may be such that switching to $\sigma_{2}$ is justified.

The previous reasoning is illustrated by a small example.

$$
\begin{gathered}
L \equiv 100 \text { (deterministic), } \\
W \in \nVdash:=\{4,104,204,1000\}, \\
P(W=w)=0.25, \quad w \in \% ; \\
\sigma_{1}=1, \quad \sigma_{2}=2, \\
c_{1}=1, \quad c_{2}=2, \quad c_{d}=10, \\
K_{d}, K_{2}>0 .
\end{gathered}
$$

This example inhibits all properties just mentioned. If $w=4$ then the system will not break down, even when $\sigma_{1}$ is applied. If $w=104$ then the system breaks down when $\sigma_{1}$ is applied, whereas the system keeps operating when $\sigma_{2}$ is applied. If $w=204$ then the system breaks down, even if $\sigma_{2}$ is applied.

Several policies are excluded from consideration beforehand. In Theorem 2.1 we have shown that the optimal switching policy at the beginning of a down period is of CLR type. So, only CLR policies are considered in state $\left(D, \sigma_{1}\right.$, $w)$. Furthermore, all policies that choose $\sigma_{2}$ in state $(U, 4)$ are nonoptimal, because a fixed cost $K_{2}$ is incurred whereas no variable cost savings are made.

For each of the remaining 20 policies we have derived explicit expressions for the average cost as function of $K_{d}$ and $K_{2}$ (using a regenerative approach, which is explained in Section 3.1). Now consider a four-region policy $\pi_{4}$, which never switches rates in a down state, and which chooses $\sigma_{1}$ in $(U, w)$ if $w \in\{4,204\}$ and $\sigma_{2}$ if $w \in\{104,1000\}$. By comparing the average cost function of $\pi_{4}$ with all other average cost functions, we conclude that $\pi_{4}$ outperforms all control limit rules if the following three conditions on $K_{d}$ and $K_{2}$ are satisfied:

$$
\begin{aligned}
& K_{d}<13026-27.08 K_{2}, \\
& K_{d}>1344-5.43 K_{2}, \\
& K_{d}>1.02 K_{2}-12 .
\end{aligned}
$$

$\pi_{4}$ is optimal if (27) is replaced by

$$
K_{d}<1344-1.90 K_{2}
$$

Since the amount of work $W$ is completely known to the repairman after inspection, its distribution function $G(w)$ does not have any influence on the form of the optimal policy. Note that in both intuitive reasoning and the example, 
the repair policy is tuned to the chance of the occurrence of a system breakdown, which in turn is determined by the lifetime distribution $F(l)$. We expect the optimality of a non-CLR in state $(U, w)$ with $K_{d}>0$ to depend strongly on the form of $F(l)$. If $F(l)$ is a continuous distribution function and if $\sigma_{L}^{2}$ (variance of $L$ ) deviates significantly from zero, then no accurate prediction of $L$ can be given. In this case the long-run average cost function will be quite insensitive to the form of the repair policy applied. So, even if a non-CLR is the optimal policy, the best CLR will be close to optimal. If $\sigma_{L}^{2}$ is close to zero then it may be possible to fine-tune the repair policy to the quite deterministic lifetimes of the units such that a non-CLR is optimal.

\section{PERFORMANCE MEASURES UNDER CLR $\left(m_{U}, m_{D}\right)$}

\subsection{Average Cost Function}

In this section we compute the long-run average cost $\mathrm{AC}\left(m_{U}, m_{D}\right)$ of controlling our two-unit standby system by a two-dimensional control limit rule $\operatorname{CLR}\left(m_{U}, m_{D}\right)$. This formula may be useful when searching for the optimal values of $m_{U}$ and $m_{D}$.

The time epochs at which one unit starts operating and an (instantaneous) inspection is carried out on the other one, are regeneration points for the system. The evolution in time of our system can be modeled by a regenerative process, i.e., after every regeneration point the system evolves as if it has just been started. The time between two regeneration points is called a cycle. From the theory of regenerative processes (cf. Tijms [11]) we know that the average cost function can be obtained from

$$
\mathrm{AC}\left(m_{U}, m_{D}\right):=\frac{E[\text { cycle cost }]}{E[\text { cycle length }]}
$$

The expected length of a cycle is obtained from

$$
E[\text { cycle length }]:=\mu_{L}+P\left(L<\frac{W}{\sigma}\right) E[\text { Down }]
$$

where the probability of a system breakdown within a cycle is given by

$$
P\left(L<\frac{W}{\sigma}\right)=\int_{0}^{m_{U}} F\left(\frac{w}{\sigma_{1}}\right) d G(w)+\int_{m_{U}}^{\infty} F\left(\frac{w}{\sigma_{2}}\right) d G(w)
$$

and

$E[$ Down $]:=$ expected length of a system down period, 
which is computed in Section 3.2. The expected cost during one cycle is computed from

$E[$ cycle cost $]:=c_{1} E\left[\right.$ time $\left.\sigma_{1}\right]+K_{2} P\left(\sigma_{2}\right.$ is chosen $)$

$$
+c_{2} E\left[\text { time } \sigma_{2}\right]+P\left(L<\frac{W}{\sigma}\right)\left(K_{d}+c_{d} E[\text { Down }]\right),
$$

where $E$ [time $\sigma_{i}$ ] denotes the expected time the repairman is working at rate $\sigma_{i}$ per cycle $(i=1,2)$.

$$
\begin{gathered}
E\left[\text { time } \sigma_{1}\right]=\int_{0}^{m_{D}} \frac{w}{\sigma_{1}} d G(w) \\
\quad+\int_{m_{l},}^{m_{l}}\left[\int_{1)}^{\left(w-m_{l}\right) \cdot \sigma_{1}} l d F(l)+\frac{w}{\sigma_{1}} \bar{F}\left(\frac{w-m_{D}}{\sigma_{1}}\right)\right] d G(w), \\
E\left[\text { time } \sigma_{2}\right]=\int_{m_{l}}^{m_{l}} \int_{0}^{\left(w-m_{l}\right) \cdot\left(\sigma_{1}\right.} \frac{w-\sigma_{1} l}{\sigma_{2}} d F(l) d G(w)+\int_{m_{l}}^{\infty} \frac{w}{\sigma_{2}} d G(w), \\
P\left(\sigma_{2} \text { is chosen }\right)=\int_{m_{D}}^{m_{U}} F\left(\frac{w-m_{D}}{\sigma_{1}}\right) d G(w)+\bar{G}\left(m_{U}\right) .
\end{gathered}
$$

It is not possible to derive a closed-form expression for $\mathrm{AC}\left(m_{U}, m_{D}\right)$. After reduction. (29) and (31) have to be evaluated using some numerical routines.

\subsection{Moments of System Up- and Downtimes}

Often long-run average measures as defined in Section 3.1 provide insufficient information about the actual way a system will be operating. For planning purposes it may be important, e.g., to be able to predict the length of an arbitrary down period. In such situations it is useful to know the moments (mean, variance, etc.) of system up- and downtimes, which are obtainable from the corresponding Laplace transforms:

$$
\begin{aligned}
& \phi_{U}(s):=\int_{0}^{\infty} e^{-s t} P(\mathrm{Up}>t) d t \\
& \phi_{D}(s):=\int_{0}^{\infty} e^{-s t} P(\text { Down }>t) d t, \quad s>0 .
\end{aligned}
$$

Furthermore $\phi_{L}(s)$ denotes the Laplace transform of the lifetime distribution function. Using the regenerative nature of our system (cf. Section 3.1), in Theorem 3.1 we present a closed-form expression of $\phi_{U}(s)$. Theorem 3.2 gives $\phi_{D}(s)$. 


\section{THEOREM 3.1:}

$$
\phi_{U}(s)=\phi_{L}(s) /[1-\gamma(s)], \quad s>0 .
$$

where

$$
\begin{gathered}
\gamma(s):=\int_{0}^{m_{\ell} \sigma_{1}} e^{-s t} G\left(\sigma_{1} t\right) d F(t)+\int_{m_{l^{\prime} \omega_{2}}}^{\infty} e^{-s t} G\left(\sigma_{2} t\right) d F(t) \\
-G\left(m_{\ell^{\prime}}\right) \int_{m_{l} \sigma_{2}}^{m_{l} \sigma_{l}} e^{-s t} d F(t) .
\end{gathered}
$$

PROOF: We consider an arbitrary regeneration cycle (cf. Section 3.1 ) to begin at time 0 with

$$
\begin{aligned}
& L:=\text { lifetime of the unit operating in that cycle, } \\
& R:=\text { repair time of the unit under repair in that cycle. }
\end{aligned}
$$

We need the conditional probability that the repair will have been completed before the operating unit fails, given that it fails at time $l$; i.e., we need the conditional repair-time distribution given that no additional opportunity to switch repair rates has occurred yet:

$$
\begin{aligned}
P(R \leq l \mid L=l)= & P\left(\frac{W}{\sigma_{1}} \leq l ; W \leq m_{U}\right)+P\left(\frac{W}{\sigma_{2}} \leq l ; W>m_{l}\right) \\
& = \begin{cases}G\left(\sigma_{1} l\right), & \text { if } 0 \leq l \leq \frac{m_{U}}{\sigma_{2}}, \\
G\left(\sigma_{1} l\right)+G\left(\sigma_{2} l\right)-G\left(m_{U}\right), & \text { if } \frac{m_{U}}{\sigma_{2}}<l \leq \frac{m_{U}}{\sigma_{1}}, \\
G\left(\sigma_{2} l\right), & \text { if } \frac{m_{U}}{\sigma_{1}}<l<x .\end{cases}
\end{aligned}
$$

Due to the regenerative nature of our system the distribution of the length of a system up period is obtained from

$$
\begin{aligned}
P(\mathrm{Up}>t) & =P(L>t)+\int_{0}^{t} P(R \leq l ; \mathrm{Up}>t-l \mid L=l) d F(l) \\
& =\bar{F}(t)+\int_{0}^{t} P(R \leq l \mid L=l) P(\mathrm{Up}>t-l) d F(l) .
\end{aligned}
$$


Taking Laplace transforms and using (33) we obtain

$$
\begin{aligned}
\phi_{l}(s) & =\phi_{L}(s)+\int_{t=11}^{\infty} e^{\cdot s} \int_{l=1 !}^{l} P(R \leq l \mid L=l) P(\mathrm{Up}>t-l) d F(l) d t \\
& =\phi_{L}(s)+\int_{l=1}^{\infty} P(R \leq l \mid L=l) e^{-s l} \int_{t=l}^{\infty} e^{-s(t-l)} P(\mathrm{Up}>t-l) d t d F(l) \\
& =\phi_{L}(s)+\phi_{U}(s) \int_{0}^{\infty} e^{-s l} P(R \leq l \mid L=l) d F(l) \\
& =\phi_{L}(s)+\phi_{L}(s) \gamma(s) .
\end{aligned}
$$

which completes the proof of Theorem 3.1 .

Let

$$
\begin{aligned}
& N(s):=\int_{0}^{m_{D} / \sigma_{1}} e^{-s t} \int_{\sigma_{1} t}^{m_{l} t} F\left(\frac{w}{\sigma_{1}}-t\right) d G(w) d t \\
& +\int_{m_{D^{\prime}} \sigma_{2}}^{m_{t} / \sigma_{2}} e^{-s t} \int_{\sigma_{2} t}^{m_{U}} F\left(\frac{w-\sigma_{2} t}{\sigma_{1}}\right) d G(w) d t \\
& +\int_{m_{l}}^{\infty} e^{-s\left(w / v_{2}\right)} \int_{0}^{w / v_{2}} e^{s u} F(u) d u d G(w) \\
& -\int_{m_{l^{\prime}} / w_{2}}^{m_{D^{\prime}} \sigma_{1}} e^{-s t} d t \int_{m_{D}}^{m_{U}} F\left(\frac{w-m_{D}}{\sigma_{1}}\right) d G(w) .
\end{aligned}
$$

Recall from Section 3.1 that the probability of a system breakdown occurring in a cycle is denoted by $P(L<W / \sigma)$, which is defined by (30). Now the Laplace transform of the length of a system down period is obtained from the following theorem.

THEOREM 3.2:

$$
\phi_{D}(s)=N(s) / P\left(L<\frac{W}{\sigma}\right) .
$$

PROOF: See Appendix C.

\subsection{Additional Long-Run Average Measures}

Several important operational characteristics can now easily be calculated using the expressions found in Sections 3.1 and 3.2. For instance: 
- $E[\mathrm{Up}] /(E[\mathrm{Up}]+E[$ Down $]):=$ availability of the system (which is the fraction of time the system is operational)

- $P(L<W / \sigma) / E[$ cycle] $:=$ mean number of system breakdowns occurring per time unit

- $1 / E[$ cycle $]:=$ mean number of repairs performed per time unit

- $\bar{G}\left(m_{U}\right):=$ fraction of the total number of repairs that are fully carried out at rate $\sigma_{2}$

- $P\left(\sigma_{2}\right.$ is chosen $)-\bar{G}\left(m_{U}\right):=$ fraction of the total number of repairs that are started at rate $\sigma_{1}$ and completed at rate $\sigma_{2}$ after an intermediate switch at system breakdown

- $E\left[\right.$ time $\left.\sigma_{i}\right] / E\left[\right.$ cycle] $:=$ fraction of time the repairman is working at rate $\sigma_{i}, i=1$, 2

- $\left(E[\right.$ cycle $]-\sum_{i=1}^{i=2} E\left[\right.$ time $\left.\sigma_{i}\right] / E[$ cycle $]:=$ fraction of time the repairman is idle

\section{CONCLUDING REMARKS}

Several questions are still open for further research. At first, for the general model, in Theorem 2.1 we have shown that in state $\left(D, \sigma_{1}, w\right)$ it is average cost optimal to switch to the fast repair rate $\sigma_{2}$ according to a CLR. For $K_{d}=0$, in Theorem 2.2, we have shown that in state $(U, w)$ the optimal repair rate is chosen according to a CLR if either $c_{1} / \sigma_{1} \geq c_{2} / \sigma_{2}$ or $F(l)$ is IFR. We have not been able to obtain structural results on the optimal policy if both $c_{1} / \sigma_{1}<$ $c_{2} / \sigma_{2}$ and $F(l)$ is non-IFR, but on the contrary we do not have intuitive arguments why a control limit rule would not be optimal in this case.

Let

$m_{U}^{G}, m_{D}^{G}, g^{G}$ : the optimal control limits, and the minimum average costs, of the general model considered in Theorems 2.1 and 2.2.

$m_{U}^{R}, g^{R} \quad:$ the optimal control limit, and the minimum average costs, of the restricted model considered in Theorem 2.3.

For $K_{d}=0$, the following relationship is intuitively clear:

$$
m_{U}^{G} \geq m_{U}^{R} \geq m_{D}^{G}
$$

After choosing the proper repair rate in the restricted model, it takes some time before the system eventually breaks down; thus $m_{U}^{R} \geq m_{D}^{G}$. This inequality follows easily from (7) and (26), since $g^{R} \geq g^{G}$. In the restricted model there is no additional opportunity to switch repair rates; thus we expect $m_{U}^{G} \geq m_{U}^{R}$. From i and ii in the proof of Theorem 2.2, it is immediately clear that $m_{U}^{G} \geq m_{D}^{G}$. However, we have not yet found the right arguments to prove the first inequality of (35).

By numerical experiments one can investigate monotonicity of the average cost function $\mathrm{AC}\left(m_{U}, m_{D}\right)$ as a function of $m_{U}$ and $m_{D}$. This result may lead to considerable savings of computation time when efficiently searching for the optimal control limits $m_{U}^{*}$ and $m_{D}^{*}$.

Most intriguing is the form of the optimal policy for $K_{d}>0$. At the end of Section 2.3 we argued that the best control limit rule will be (close to) optimal if $\sigma_{L}^{2}$ deviates significantly from zero. This conjecture may be verified by numerical experiments after properly discretizing the probability distribution functions. Other questions may be answered, such as: When is the best CLR a good 
alternative to an optimal non-CLR? What is the influence of $K_{d}$ and $F(l)$ on the form of the optimal policy? Are there optimal policies with more than four regions? (we believe not), etc.

Sensitivity results may be obtained by varying input parameters during the numerical experiments. One might, for instance, vary the fast repair rate, the slow repair rate, variable down costs (loss of production), or one might apply a nonoptimal control limit rule, etc.

\section{APPENDIX A. EXISTENCE OF AN AVERAGE COST OPTIMAL POLICY}

In this appendix we outline the proof of the existence of an average cost optimal policy, under the additional assumption that $G(w)$ has a finite and $F(l)$ has an infinite support. First we make use of Theorem 2 of Ross [10], which guarantees the existence of an average cost optimal policy, provided the following two conditions are satisfied.

CONDITION A.1: There exist finite numbers $\delta>0$ and $\epsilon>0$ such that

$$
P(T(x ; \sigma) \leq \delta)<1-\epsilon, \quad \text { for all } x \in \mathscr{x}, \quad \sigma \in\left\{\sigma_{1}, \sigma_{2}\right\},
$$

where $T(x ; \sigma)$ denotes the time until the next decision epoch, given the current state $x$ and current action $\sigma$.

CONDITION A.2: There exists a bounded Baire function $v(\cdot)$ on $\{$ and a constant $g$ satisfying the optimality equation (3).

Theorem 2 of Ross [10] states that under Conditions A.1 and A.2 any policy which, when in state $x$, selects an action minimizing the right-hand side of (3) is average cost optimal.

Condition A.1, however, is not satisfied in our model, since for $i=1,2$

$$
\inf _{x \in i}\left\{\tau\left(x ; \sigma_{i}\right)\right\}=0 .
$$

To overcome this difficulty, we slightly modify our original semi-Markov decision process into an equivalent semi-Markov decision process for which Condition A.1 does hold. This modification is based on a preliminary analysis of the average cost optimality equations (4)-(6). Note that (36) is caused by those states $x \in I$ which are represented by $\left(D, \sigma_{i}, w\right)$ for small values of $w(i=1$, $2)$. Hence we modify our semi-Markov decision process such that those states are removed from $x$. Due to $(6)$ the states $\left(D, \sigma_{2}, w\right)$ can be removed without any difficulty for all $w \geq 0$ : Simply insert (6) into the second term of (4) [cf. (9)]. Moreover, we note that $\sigma_{1}$ is the minimizing action in (5) (independently of the value of $g \geq 0)$ in those states $\left(D, \sigma_{1}, w\right)$, with $w \leq w^{*}$, where

$$
w^{*}:=\frac{\sigma_{1} K_{2}}{c_{1}+c_{d}}>0 \text {. }
$$


So by removing those states from the state space for which the optimal action can be determined beforehand, we arrive at the following modified semi-Markov decision process, which is equivalent to the original one (i.e., the average cost optimality equations for both models have exactly the same solutions):

$$
\begin{aligned}
& x_{m}=\{(U, w) ; w \geq 0\} \cup\left\{\left(D, \sigma_{1}, w\right) ; w \geq w^{*}\right\}, \\
& A_{m}=A_{m}(x)=\left\{\sigma_{1}, \sigma_{2}\right\}, \quad x \in x_{m}^{\prime}, \\
& \tau_{m}\left(U, w ; \sigma_{1}\right)=\mu_{L}+\int_{0}^{w / \sigma_{1}} F(t) d t, \quad 0 \leq w \leq w^{*}, \\
& \tau_{m}\left(U, w ; \sigma_{1}\right)=\mu_{L}+\int_{\left(w-w^{*}\right) / \sigma_{1}}^{w / \sigma_{1}}\left\{F(t)-F\left(\frac{w-w^{*}}{\sigma_{1}}\right)\right\} d t, \quad w>w^{*} \\
& \tau_{m}\left(U, w ; \sigma_{2}\right)=\mu_{L}+\int_{0}^{w / \sigma_{2}} F(t) d t, \quad w \geq 0 \\
& \tau_{m}\left(D, \sigma_{1}, w ; \sigma_{i}\right)=\frac{w}{\sigma_{i}}, \quad w \geq w^{*}, \quad i=1,2, \\
& c_{m}\left(U, w ; \sigma_{1}\right)=\frac{c_{1} w}{\sigma_{1}}+K_{d} F\left(\frac{w}{\sigma_{1}}\right)+c_{d} \int_{0}^{w / \sigma_{1}} F(t) d t, \quad 0 \leq w \leq w^{*}, \\
& c_{m}\left(U, w ; \sigma_{1}\right)=c_{1}\left\{\int_{0}^{w / \sigma_{1}}[1-F(t)] d t\right. \\
& \left.+\int_{\left(w-w^{*}\right) / \sigma_{1}}^{w / \sigma_{1}}\left[F(t)-F\left(\frac{w-w^{*}}{\sigma_{1}}\right)\right] d t\right\} \\
& +c_{d} \int_{\left(w-w^{*} / \sigma_{1}\right.}^{w / \sigma_{1}}\left[F(t)-F\left(\frac{w-w^{*}}{\sigma_{1}}\right)\right] d t \\
& +K_{d}\left[F\left(\frac{w}{\sigma_{1}}\right)-F\left(\frac{w-w^{*}}{\sigma_{1}}\right)\right], \quad w>w^{*} \\
& c_{m}\left(U, w ; \sigma_{2}\right)=K_{2}+\frac{c_{2} w}{\sigma_{2}}+K_{d} F\left(\frac{w}{\sigma_{2}}\right)+c_{d} \int_{0}^{w / \sigma_{2}} F(t) d t, \quad w \geq 0 \\
& c_{m}\left(D, \sigma_{1}, w ; \sigma_{1}\right)=K_{d}+\left(c_{1}+c_{d}\right) \frac{w}{\sigma_{1}}, \quad w \geq w^{*}, \\
& c_{m}\left(D, \sigma_{1}, w ; \sigma_{2}\right)=K_{d}+K_{2}+\left(c_{2}+c_{d}\right) \frac{w}{\sigma_{2}}, \quad w \geq w^{*} .
\end{aligned}
$$


Finally, the one-step transition probabilities are given by

$$
\begin{gathered}
p_{m}\left\{(U,[0, v]) \mid\left(U, w ; \sigma_{1}\right)\right\}=G(v), \quad 0 \leq w \leq w^{*}, \\
p_{m}\left\{(U,[0, v]) \mid\left(U, w ; \sigma_{1}\right)\right\}=G(v)\left[1-F\left(\frac{w-w^{*}}{\sigma_{1}}\right)\right], \quad w \geq w^{*}, \\
p_{m}\left\{(U,[0, v]) \mid\left(U, w ; \sigma_{2}\right)\right\}=G(v), \quad w \geq 0, \\
p_{m}\left\{\left(D, \sigma_{1},\left[w-\sigma_{1} t, w\right]\right) \mid\left(U, w ; \sigma_{1}\right)\right\}=F(t), \quad w \geq w^{*}, 0 \leq t<\frac{w-w^{*}}{\sigma_{1}}, \\
p_{m}\left\{(U,[0, v]) \mid\left(D, \sigma_{1}, w ; \sigma_{i}\right)\right\}=G(v), \quad w \geq w^{*}, \quad i=1,2 .
\end{gathered}
$$

Note that for this modified semi-Markov decision process the transition times $T_{m}(x ; \sigma)$ between two successive decision epochs have the property

$$
T_{m}(x ; \sigma) \geq \min \left\{L, \frac{w^{*}}{\sigma_{2}}\right\}, \quad \text { for all } x, \sigma
$$

Since $F(0)=0$, Condition A.1 is certainly satisfied for the modified model. To verify Condition A.2 for the modified model, we invoke Theorem 3.1 in Kurano [8], which states that Condition A.2 holds if Conditions A. 3 and A.4 below are fulfilled.

CONDITION A.3: The one-step transition functions $\tau_{m}(x ; \sigma)$ and the onestep cost functions $c_{m}(x ; \sigma)$ are bounded on $\mathscr{x}_{m}^{\prime} \times A_{m}$.

CONDITION A.4: There exists a finite measure $\gamma$ on $x_{m}$ and a $0<\beta<1$ such that

$$
\begin{gathered}
p_{m}\{\mathscr{R} \mid(x ; \sigma)\}>\tau_{m}(x ; \sigma) \gamma(\mathscr{R}), \quad \text { for any Borel set } \mathscr{R} \text { of } \mathscr{X}_{m}^{\prime}, \\
\gamma\left(\mathscr{X}_{m}^{\prime}\right)>(1-\beta) / \tau_{m}(x ; \sigma), \quad \text { for any }(x ; \sigma) \in \mathscr{X}_{m}^{\prime} \times A_{m} .
\end{gathered}
$$

Condition A.3 is trivially satisfied under the following assumption.

ASSUMPTION 5: $G(w)$ has finite support $\left[0, w_{\max }\right]$ and $F(l)$ has infinite support.

Note that Assumption 5 implies that the state space $\mathscr{x}_{m}$ can be restricted to the states $(U, w)$ and $\left(D, \sigma_{1}, w\right)$ with $w \leq w_{\max }$.

From the specification of the one-step transition functions $\tau_{m}(x ; \sigma)$ and the one-step transition probabilities $p_{m}\{\cdot \mid(x ; \sigma)\}$ it is straightforwardly verified that 
the following choices for the measure $\gamma(\cdot)$ and the number $\beta$ satisfy Condition A.4:

$$
\begin{aligned}
\gamma(U,[0, v]) & =\frac{G(v)\left[1-F\left(\frac{w_{\max }}{\sigma_{1}}\right)\right]}{\tau_{\max }}, \quad v \geq 0, \\
\gamma\left(D, \sigma_{1},[0, w]\right) & =0, \quad w \geq 0,
\end{aligned}
$$

and

$$
1-\beta=\frac{\tau_{\min }}{\tau_{\max }}
$$

where

$$
\tau_{\min }:=\inf _{x, \sigma}\{\tau(x ; \sigma)\} \quad \text { and } \quad \tau_{\max }:=\sup _{x, \sigma}\{\tau(x ; \sigma)\}
$$

The exposition above yields the following theorem.

THEOREM A.1: Under Assumption 5 Eq. (3) has a bounded solution, and any policy which, when in state $x$, selects an action minimizing the right-hand side of (3) is average cost optimal.

\section{APPENDIX B. AUXILIARY LEMMAS TO THEOREM 2.2 AND 2.3}

Let $H(w)$ be defined by (17).

LEMMA B.1: If $F(l)$ is IFR then $H(w)$ is unimodal.

PROOF: Since $H(w)=0$ if $F\left(w / \sigma_{2}\right)=1$, and $H(w)$ is monotonically decreasing if $F\left(w / \sigma_{2}\right)<1$ and $F\left(w / \sigma_{1}\right)=1$, we only consider $w$ such that $F\left(w / \sigma_{1}\right)<1$. From (17) and (21) we note that

$$
H(0)=0 ; \quad H^{\prime}(0)>0 ; \quad H(\infty)=0 .
$$

So, there is at least one solution to the following equation:

$$
H^{\prime}(w)=0 .
$$

$H(w)$ is unimodal iff (37) has a unique solution. For convenience we assume

$$
\sigma_{1}=1 ; \quad \sigma_{2}>1
$$


Now (37) is equivalent with

$$
Q(w):=\frac{1-F(w)}{1-F\left(w / \sigma_{2}\right)}=\frac{1}{\sigma_{2}} .
$$

Since

$$
Q(0)=1>\frac{1}{\sigma_{2}}
$$

a solution to (38) is unique if

$$
Q^{\prime}(w)<0 \Leftrightarrow \frac{1}{\sigma_{2}} \frac{f\left(w / \sigma_{2}\right)}{\left[1-F\left(w / \sigma_{2}\right)\right]}<\frac{f(w)}{1-F(w)},
$$

which is satisfied if $F(l)$ is IFR.

LEMMA B.2: $\quad H(\alpha w) \geq \alpha H(w), w \geq 0 ; 0 \leq \alpha \leq 1$.

PROOF:

$$
\begin{aligned}
\alpha H(w) & =\int_{w / \sigma_{2}}^{w / \sigma_{1}} \alpha[1-F(t)] d t \\
& \leq \int_{w / \sigma_{2}}^{w / \sigma_{2}+\alpha\left(w / \sigma_{1}-w / \sigma_{2}\right)}[1-F(t)] d t \\
& \leq \int_{\alpha w / \sigma_{2}}^{\alpha w / \sigma_{1}}[1-F(t)] d t=H(\alpha w) .
\end{aligned}
$$

REMARK B.1: Note that Lemma B.2 is equivalent with the statement that

$$
\frac{H(w)}{w} \text { is nonincreasing for } w \geq 0
$$

In the literature this property is referred to as star-shapedness of $-H(w)$. For a discussion of the relation between star-shapedness and the IFRA property of distribution functions we refer to Barlow and Proschan [2]. Note, however, that Lemma B. 2 does not require any structure on the probability distribution function $F(l)$. 


\section{APPENDIX C. PROOF OF THEOREM 3.2: $\phi_{D}(s)$}

Consider an arbitrary regeneration cycle to begin at time 0 . Let

$$
\begin{aligned}
\sigma & :=\text { initial repair rate } \\
\sigma^{\prime} & :=\text { repair rate after system breakdown. }
\end{aligned}
$$

The distribution of the length of a system down period is given by

$$
\begin{aligned}
P(\text { Down }>t) & =P\left(\frac{W-\sigma L}{\sigma^{\prime}}>t \mid L<\frac{W}{\sigma}\right) \\
& =P\left(\frac{W-\sigma L}{\sigma^{\prime}}>t\right) / P\left(L<\frac{W}{\sigma}\right) .
\end{aligned}
$$

$P(L<W / \sigma)$ is obtained from (30). Let the indicator function $I(a>b)$ with $a$, $b \in \mathbb{R}$ be defined by

$$
I(a>b)= \begin{cases}1, & \text { if } a>b(\text { true }) \\ 0, & \text { otherwise (false) }\end{cases}
$$

Now

$$
\begin{aligned}
P\left(\frac{W-\sigma L}{\sigma^{\prime}}>t\right)= & \int_{0}^{m_{U}} P\left(\frac{w-\sigma_{1} L}{\sigma^{\prime}}>t\right) d G(w) \\
& +\int_{m_{U}}^{\infty} P\left(\frac{w-\sigma_{2} L}{\sigma_{2}}>t\right) d G(w) \\
= & A(t)+B(t)+C(t),
\end{aligned}
$$

where $A(t), B(t)$, and $C(t)$ are defined by

$$
\begin{aligned}
& A(t):=\int_{0}^{m_{U}} \int_{\left(w-m_{D}\right) / \sigma_{l}}^{w / \sigma_{1}} I\left(\frac{w}{\sigma_{1}}-l>t\right) d F(l) d G(w), \\
& B(t):=\int_{m_{D}}^{m_{U}} \int_{0}^{\left(w-m_{D}\right) / \sigma_{1}} I\left(\frac{w-\sigma_{1} l}{\sigma_{2}}>t\right) d F(l) d G(w), \\
& C(t):=\int_{m_{U}}^{\infty} P\left(L<\frac{w}{\sigma_{2}}-t\right) d G(w) .
\end{aligned}
$$


Careful inspection of $A(t), B(t)$, and $C(t)$ leads to the following expressions:

$$
\begin{aligned}
& A(t)= \begin{cases}\int_{\sigma_{1} t}^{m_{U}} F\left(\frac{w}{\sigma_{1}}-t\right) d G(w)-\int_{m_{D}}^{m_{U}} F\left(\frac{w-m_{D}}{\sigma_{1}}\right) d G(w), & \text { if } 0 \leq t<\frac{m_{U}}{\sigma_{1}}, \\
0, & \text { if } t \geq \frac{m_{U}}{\sigma_{1}},\end{cases} \\
& B(t)= \begin{cases}\int_{m_{D}}^{m_{U}} F\left(\frac{w-m_{D}}{\sigma_{1}}\right) d G(w), & \text { if } 0 \leq t<\frac{m_{D}}{\sigma_{2}}, \\
\int_{\sigma_{2} t}^{m_{U}} F\left(\frac{w-\sigma_{2} t}{\sigma_{1}}\right) d G(w), & \text { if } \frac{m_{D}}{\sigma_{2}} \leq t<\frac{m_{U}}{\sigma_{2}}, \\
0, & \text { if } t \geq \frac{m_{U}}{\sigma_{2}},\end{cases} \\
& C(t)= \begin{cases}\int_{m_{U}}^{\infty} F\left(\frac{w}{\sigma_{2}}-t\right) d G(w), & \text { if } 0 \leq t<\frac{m_{U}}{\sigma_{2}}, \\
\int_{\sigma_{2} t}^{\infty} F\left(\frac{w}{\sigma_{2}}-t\right) d G(w), & \text { if } t \geq \frac{m_{U}}{\sigma_{2}} .\end{cases}
\end{aligned}
$$

$\phi_{D}(s)$ can now be obtained by integrating over the appropriate regions for $t$ :

$$
\phi_{D}(s)=\int_{0}^{\infty} e^{-s t}[A(t)+B(t)+C(t)] d t / P\left(L<\frac{W}{\sigma}\right)
$$

which gives (34).

\section{REFERENCES}

[1] Albright, S.C., "Optimal Maintenance-Repair Policies for the Machine Repair Problem," Naval Research Logistics Quarterly, 27, 17-27 (1980).

[2] Barlow, R.E., and Proschan, F., Statistical Theory of Reliability and Life Testing, Holt, Rinehart and Winston, New York, 1975.

[3] Cho, D.I., and Parlar, M., "A Survey of Maintenance Models for Multi-Unit Systems," European Journal of Operational Research, 51, 1-23 (1991).

[4] Crabill, T.B., "Optimal Control of a Maintenance System with Variable Service Rates," Operations Research, 22, 736-745 (1974).

[5] Hatoyama, Y., "Markov Maintenance Models with Control of Queue," Journal of the Operations Research Society of Japan, 20(3), 164-181 (1977).

[6] Karmeshu, and Jaiswal, N.K., "A Machine Interference Model with Threshold Effect," Journal of Applied Probability, 18, 491-498 (1981).

[7] Kawai, H. "An Optimal Maintenance Policy of a Two-Unit Standby System," Transactions of the IECE of Japan, E64(9), 579-582 (1981).

[8] Kurano, M., "Semi-Markov Decision Processes and their Applications in Replacement Models," Journal of the Operations Research Society of Japan, 28( 1) , 18-29 (1985).

[9] Pierskalla, W.P., and Voelker, J.A., "A Survey of Maintenance Models: The Control and Surveillance of Deteriorating Systems," Naval Research Logistics Quarterly, 23, 353-388 (1976). 
[10] Ross, S.M., “Average Cost Semi-Markov Decision Processes. Journal of Applied Probability, 7, 649-656 (1970).

[11] Tijms, H.C., Stochastic Modelling and Analysis: A Computational Approach, Wiley, Chichester, 1986.

[12] Weber, R.R., and Stidham, S., Jr., "Optimal Control of Service Rates in Networks of Queues," Advances in Applied Probability, 19, 202-218 (1987).

[13] Winston, W., "Optimal Control of Discrete and Continuous Time Maintenance Systems with Variable Service Rates," Operations Research, 25(2), 259-268 (1977).

[14] Winston, W., "Optimality of Monotonic Policies for Multiple-Server Exponential Queueing Systems with State-Dependent Arrival Rates," Operations Research, 26(6), 1089-1094 (1978).

Manuscript received July 7, 1992

Revised manuscript received November 4, 1992

Accepted November 17, 1992 